\title{
L'écrit documentaire médiéval et le projet des Plus anciens documents linguistiques de la France
}

\author{
Glessgen, Martin-Dietrich
}

\begin{abstract}
L'écrit documentaire médiéval comporte des textes produits par l'administration, le commerce et la pratique judiciaire ainsi que la production épistolaire. Son volume dépasse de très loin celui de tous les autres genres textuels confondus et il comporte de riches renseignements dans tous les domaines du langage. L'état d'étude philologique et linguistique de ce patrimoine est toutefois largement insuffisant pour toute la Romania. Dans le domaine gallo-roman, le projet centenaire des Plus anciens documents linguistiques de la France est paradigmatique dans sa tentative de répertorier, d'éditer et de préparer à l'analyse linguistique les témoins médiévaux dans une logique systématique. Les différentes étapes du projet, fondé par Paul Meyer et Clovis Brunel, continué par Jacques Monfrin et dirigé actuellement par Martin Glessgen sous les auspices de la philologie électronique montrent autant le potentiel des textes documentaires que les contraintes intrinsèques à leur étude.
\end{abstract}

Posted at the Zurich Open Repository and Archive, University of Zurich

ZORA URL: https://doi.org/10.5167/uzh-134203

Book Section

Published Version

Originally published at:

Glessgen, Martin-Dietrich (2015). L'écrit documentaire médiéval et le projet des Plus anciens documents linguistiques de la France. In: Trotter, David. Manuals of Romance Linguistics. Berlin/Boston: De Gruyter, 258-286. 


\title{
11 L'écrit documentaire médiéval et le projet des Plus anciens documents linguistiques de la France
}

\begin{abstract}
L'écrit documentaire médiéval comporte des textes produits par l'administration, le commerce et la pratique judiciaire ainsi que la production épistolaire. Son volume dépasse de très loin celui de tous les autres genres textuels confondus et il comporte de riches renseignements dans tous les domaines du langage. L'état d'étude philologique et linguistique de ce patrimoine est toutefois largement insuffisant pour toute la Romania. Dans le domaine gallo-roman, le projet centenaire des Plus anciens documents linguistiques de la France est paradigmatique dans sa tentative de répertorier, d'éditer et de préparer à l'analyse linguistique les témoins médiévaux dans une logique systématique. Les différentes étapes du projet, fondé par Paul Meyer et Clovis Brunel, continué par Jacques Monfrin et dirigé actuellement par Martin Glessgen sous les auspices de la philologie électronique montrent autant le potentiel des textes documentaires que les contraintes intrinsèques à leur étude.
\end{abstract}

Keywords : scriptae, chartes, philologie informatique, textes administratifs

\section{L'écrit documentaire dans l'histoire de l'écrit et en linguistique historique}

1.1 L'écrit documentaire est présent dès les premiers textes rédigés il y a cinq millénaires en Mésopotamie et en Égypte (cf. HSK 10, art. 18s.). À travers toute l'histoire de la scripturalité, il a occupé de loin la première place parmi les différents genres textuels par son importance quantitative. Dans la Romania médiévale, l'écrit documentaire vernaculaire se développe surtout aux XIII $/ \mathrm{XV}^{\mathrm{e}}$ siècles, investissant peu à peu la place du latin, dont les actes se sont multipliés depuis la Réforme carolingienne. Pour les deux siècles compris entre 1300 et 1500, il faut compter plusieurs centaines de millions de pages écrites en français, italien, occitan, catalan, espagnol ou en gallégo-portugais (Glessgen 2001a, 268s.).

Il s'agit, certes, de sources de type sériel, mais avec une réelle diversité interne de formes linguistiques selon les genres textuels. L'écrit documentaire comprend ainsi tous les textes produits par la gestion princière, ecclésiastique ou urbaine, par le commerce et par la pratique judiciaire, notamment : 
- $\quad$ toute forme de listes ou d'inventaires (de personnes, de terrains, de biens meubles) qui interviennent notamment dans la gestion foncière (censiers etc.) ou commerciale (inventaires de marchandises) ;

- $\quad$ toute forme de documents comptables (surtout des registres de recettes et dépenses qui sont souvent très riches d'un point de vue lexical, notamment en contexte princier) ;

- $\quad$ tout acte de la pratique de droit (les chartes, souvent transmises par des cartulaires, traitant tout genre de questions liées au droit privé : ventes et donations, règlements de litiges et arbitrages, inféodations, etc.; plus tard, les registres princiers ou urbains réunissant des pétitions ou demandes, des décrets ou privilèges, des comptes rendus de décisions en cas de litige) ;

- $\quad$ toujours dans le cadre du droit, certains genres bien définis qui sont plus proches de la pratique que de la théorie juridique (testaments, sentences judiciaires ou encore des statuts, des chartes-loi et, dans une moindre mesure, des coutumes); la théorie juridique, quant à elle, s'intègre plutôt dans les traditions textuelles d'un savoir spécialisé, même si elle se trouve en interaction avec l'écrit documentaire (cf. Kabatek 2005, 124-130);

- $\quad$ enfin, un nombre très considérable de lettres (de type politique, administratif, commerciales, privées) ; le cas le plus spectaculaire étant les ca 125.000 lettres des archives Datini (cf. Hayez 2005).

D'un point de vue interne, l'écrit documentaire fournit toutefois de nombreuses informations sur l'état et l'évolution des idiomes vernaculaires médiévaux. Dans le domaine grapho-phonétique, la précision et la densité chronologique et géolinguistique des apports dépasse de loin tous les autres genres textuels, ce qui explique sa place de choix en scriptologie. Le marquage nominal et verbal est également bien représenté. En syntaxe, seuls les textes argumentatifs comme les chartes et les lettres sont pertinents - mais il s'agit là d'ensembles importants avec un haut degré de complexité syntaxique. Les chartes permettent également de mieux cerner les tensions entre l'oralité et la scripturalité, puisque ces textes sont placés dans un environnement pragmatique et fortement médialisé. Enfin, les différents genres documentaires livrent des informations lexicales sur des champs sémantiques divers: les multiples objets du commerce et de la vie quotidienne dans les registres commerciaux, princiers et urbains, les données du monde agricole dans les chartes et la terminologie juridique dans les textes de la pratique judiciaire. S’ajoutent de nombreux noms de personnes et, surtout, de lieux, non atteignables pour l'essentiel dans les autres genres textuels. Si tout genre connaît des restrictions linguistiques et ne reflète que partiellement la diversité d'une langue historique, les genres documentaires couvrent des pans plus larges que les textes littéraires profanes, les textes religieux ou les textes relevant de savoirs spécialisés et ils ont la qualité particulière de décrire plus en détail le monde médiéval des objets.

1.2 Malgré leur richesse indéniable, les textes documentaires sont l'ensemble textuel de loin le moins étudié. Le contraste devient particulièrement saisissant si l'on compare avec la littérature (profane) dont le volume est inférieur au moins par un facteur 100, mais pour lequel le nombre d'études et de chercheurs spécialisés est bien 
plus important (cf. Carles/Glessgen 2015). Curieusement, la philologie de la seconde moitié $\mathrm{du} \mathrm{XIX}^{\mathrm{e}}$ siècle traitait d'une manière plus équitable que celle du $\mathrm{XX}^{\mathrm{e}}$ siècle ces différents genres textuels. Le XX ${ }^{\mathrm{e}}$ siècle a connu en effet une concentration croissante sur des textes littéraires, renforcé par la dé-philologisation de la linguistique depuis les années 1960. Or, la philologie littéraire est, par définition, absente des études sur les textes documentaires, alors qu'elle reste ouverte aux textes historiographiques, religieux ou même scientifiques.

Ce décalage représente un des grands paradoxes de l'historiographie linguistique et ne pourra être résorbé que par de nombreuses années de recherche. Les genres documentaires ne sont pas un sous-ensemble circonscrit, mais un univers multiforme où presque tout reste à découvrir. La diversité interne de ces ensembles textuels et leur volume rend toute tentative d'aperçu global difficile. Il existe toutefois un certain nombre de tentatives pour mieux cerner l'écrit documentaire médiéval :

- adoptant une approche régionale (= toutes les sources non-littéraires d'une région à une époque donnée) : pour l'Italie du Nord, P. Videsott a réuni pour l'époque comprise entre $c a$ 1280 et 1525 un peu plus de 2000 textes vernaculaires surtout de type documentaire pour lesquels nous disposons d'une édition, permettant ainsi un aperçu géolinguistique, chronologique et quantitatif des genres accessibles pour une région définie de la Romania médiévale (Videsott 2009, 64-237 ; une trentaine de textes se place entre $c a 1200$ et $c a 1280$ ) ;

- d'autres adoptent une approche par genres textuels (diachronique ou comparatiste) : dans la lignée notamment de W. Raible (cf. HSK 10, art. 1, la collection des ScriptOralia, etc.), différentes traditions textuelles particulièrement caractéristiques ont été étudiées de manière interprétative, par exemple les sentences judiciaires en français (Krefeld 1985), les lettres en italien (Koch 1987), le phénomène des listes (Koch 1990) ou les textes romans liés au droit romain (Kabatek 2005, 87-112) ;

- $\quad$ on relève également des approches lexicales (par définition plus partielles) : par ex. dans le domaine commercial, les études de F. Melis (1962) sur le vocabulaire contenu dans les lettres des archives Datini ou la thèse de M. Höfler (1967) sur les dénominations des tissus d'après les sources documentaires en français ;

- enfin, on mentionnera les approches grapho-phonétiques (et morphologiques) de la scriptologie qui repose traditionnellement surtout sur des textes documentaires (cf. pour le territoire de la France, Gossen 1967 ; Goebl 1970 ; Dees 1980 et la synthèse Glessgen 2012).

Mais devant l'immensité de la matière, nos connaissances sur le rôle de l'écrit documentaire dans la pratique de l'écrit et dans l'élaboration des langues romanes restent encore très partielles. Le constat est d'ailleurs bien plus flagrant encore pour les textes en latin médiéval, qui contiennent de nombreux éléments lexicaux et onomastiques vernaculaires et qui sont également significatifs pour des interrogations grapho-phonétiques, morphologiques ou syntaxiques. 


\section{Le projet des Documents linguistiques de la France (DocLing)}

Parmi les études philologiques portant sur des textes documentaires, l'entreprise des Documents linguistiques de la France est sans doute celle avec la plus grande longévité : elle a débuté à la fin du XIX ${ }^{\mathrm{e}}$ siècle et a connu un renouveau au début du XXI siècle. L'évolution du projet à travers les décennies montre ainsi certaines tendances plus générales dans le travail philologique. Le caractère prototypique ressort également dans les choix de contenu :

- avec des textes documentaires choisis, le projet est consacré à un ensemble textuel peu connu et riche pour l'analyse de tous les domaines du langage ;

- il entend documenter les débuts du genre étudié en langue vernaculaire, ce qui est particulièrement important parce que les traditions discursives connaissent une grande constance dans les choix de structure textuelle, de syntaxe et de lexique à travers les siècles(cette constance admet naturellement des transformations ; citons l'élaboration graphématique, terminologique et syntaxique des chartes et leur professionalisation accrue dans les décennies suivant 1280, cf. Monfrin 1968, 45sq. et Grübl 2014, 220sq.) ;

- il entend couvrir par une approche globalisante l'intégralité d'un territoire linguistique ;

- ses dimensions quantitatives sont suffisantes pour permettre des observations générales.

Les Documents linguistiques fournissent un corpus textuel significatif permettant des analyses philologiques et linguistiques de type exemplaire. Même s'il ne s'agit que d'une parcelle définie de l'univers documentaire, son élaboration pourrait servir à mieux planifier et structurer d'autres entreprises consacrées à d'autres langues, d'autres genres et/ou d'autres époques. Dans cette optique, l'aperçu suivant distingue les quatre phases de l'évolution du projet des DocLing, les aspects de linguistique philologique dans sa conception actuelle et les aspects de technologie informatique dans le projet actuel, qui sont a priori indépendants de la nature du genre textuel étudié.

\section{L'évolution du projet des DocLing}

\subsection{Paul Meyer}

Les quatre phases dans le projet des DocLing sont intimement liées aux différents protagonistes, à leurs choix et, bien entendu, aux divers paramètres conditionnant le travail scientifique. Paul Meyer (1840-1917) ouvrit la voie de ce grand chantier à tâtons, en sondant et déblayant un terrain parfaitement vierge. Il décrit lui-même, vers la fin de sa vie, son cheminement avec toute la clarté et précision qui le caractérisaient : 
«J'étais encore sur les bancs de l'École des chartes, que je copiais toutes les chartes provençales auxquelles je pouvais avoir accès. C'est à l'aide du recueil, bien insuffisant, que je m'étais formé et des textes imprimés, encore peu nombreux il y a trente ou quarante ans, que je rédigeai [en 1874 un] essai sur la langue d'oc et ses dialectes [...] [,] trop incomplet pour mériter de voir le jour. J'avais dû me résigner à mettre en œuvre des éléments trop peu nombreux. [...] J’ai donc reconnu de très bonne heure la nécessité d'une exploration méthodique des archives du midi de la France. Mais [...] [c]'est seulement depuis une dizaine d'années que j'ai pu consacrer à des recherches dans les archives de la France méridionale une partie de mes vacances.

Ces recherches sont souvent pénibles. [...] j'ai eu le plus ordinairement à poursuivre mes recherches dans les archives communales, qui, en bien des villes, ne sont ni classées, ni inventoriées » (Meyer 1909, I-III).

La publication du volume des Documents linguistiques du Midi de la France en 1909 fut donc l'achèvement d'un travail de longue haleine, ponctué de tentatives préliminaires, de réflexions méthodologiques et de sondages divers. Paul Meyer nourrissait ses pensées organisatrices d'un travail dans les archives et dans les bibliothèques, mais également par le recensement des travaux de ses contemporains dans ses très nombreux comptes rendus. Diverses publications de textes documentaires accompagnèrent cette longue préparation :

- dès 1874/1876, l'intégration d'une série de textes documentaires, notamment des chartes [pp. 158-173] et coutumes [pp. 173-192], dans la partie 'provençale' [p. 23-192] de son Recueil d'ancien textes bas-latins, provençaux et français - alors que la partie française [pp. 193-384] réunit comme la 'provençale' des textes littéraires religieux et profanes, mais aucun texte documentaire ;

- l'édition d'une série de documents dans le cadre de la brève étude Le langage de Die au XIII siècle (1891) [16 pp.] ;

- une publication partielle, mais déjà importante des Documents linguistiques des BassesAlpes (1898a) [104 pp.] ;

- l'édition Le livre-journal de maitre Ugo Teralh : notaire et drapier à Forcalquier (1330-1332) (1898b) [42 pp.] ;

- l'édition des Fragments du grand livre d'un drapier de Lyon (1320-1323) (1906) [17 pp.].

Le volume publié en 1909 marque une véritable rupture par rapport à ces travaux, ne serait-ce que par son volume considérable [655 pp.], mais également par le caractère systématique de l'entreprise. Il faut considérer séparément la partie des DocLing concernant l'Ain (fruit d'un autre auteur et consacré à une autre langue) et celle concernant les trois départements alpins, intégralement préparée par Paul Meyer. En parcourant les différents dépôts d'archives, il transcrivait sur place les documents qui lui semblaient les plus significatifs, en couvrant toute la période de l'écrit occitan, allant parfois jusqu'au XVII ${ }^{\mathrm{e}}$ siècle. La partie concernant les Basses-Alpes (aujourd'hui Alpes-de-Haute-Provence) est de loin la plus volumineuse avec 250 pages [pp. 169-418], et c'est également la plus équilibrée. Pour les Hautes-Alpes « les textes de langue sont relativement rares » [p. 420], ce qui explique la taille réduite de cette partie comportant à peine 60 pages [419-480]. Dans le département des AlpesMaritimes [pp.481-654], Paul Meyer s'est heurté à une situation archivistique très 
complexe, ce qui explique certains déséquilibres, notamment concernant la ville de Nice [pp. 619-634], pour laquelle tout témoignage vernaculaire fait défaut entre 1115 et 1445 .

Les grandes qualités de l'ouvrage résident dans la variété des genres documentaires réunis (inventaires divers, registres de recettes et dépenses et de délibérations municipales, chartes, coutumes) ainsi que dans la couverture chronologique et géographique qu'il fournit pour une région bien déterminée. Il s'agit d'un choix représentatif, notamment pour le département des Basses-Alpes. Les notes linguistiques et toponymiques sont très soignées, et nous avons eu loisir de nous convaincre que les glossaires comportent les lexèmes les plus significatifs.

La partie consacrée au département de l'Ain mérite une attention particulière ; cet ensemble, relativement volumineux [pp.1-166] et particulièrement soigné, fut intégralement préparé par un élève de Paul Meyer, Édouard Philipon (1851-1926). Après sa thèse de l'École des chartes sur Le dialecte du Lyonnais aux XIII e et XIVe siècles (1874), il entreprit une carrière juridique et politique et devint député de l'Ain pendant plus d'une décennie (1885-1898), tout en restant philologue (il rédigea, entre autres, le Dictionnaire topographique du département de l'Ain 1911). Ici, les éditions s'accompagnent d'analyses grapho-phonétiques [pp. 105-128; 140-148; 153-156] et d'observations sur la littérature dialectale. La combinaison entre département francoprovençal et départements occitans reste implicite, sachant que l'intitulé («Midi de la France ») se prête mal à un département situé au nord de Lyon. Par ailleurs, les textes de l'Ain comportent de nombreux éléments grapho-phonétiques francoprovençaux, mais la scripta reste majoritairement de type oïlique, comme cela est fréquent en domaine francoprovençal.

De cette réalisation impressionnante, il ressort que Paul Meyer a clairement sousestimé l'importance de son entreprise : il a traité personnellement trois départements avec une tradition de l'écrit plutôt restreinte, mais sans donner d'analyses linguistiques, à l'exception des trois glossaires (qui n'ont pas été réunis). L'utilisation de ce volume assez éclectique n'est pas aisée et cela a sans doute contribué au fait qu'il n'ait jamais donné lieu à d'importantes études lexicologiques, grapho-phonétiques et grammaticales : le FEW cite très systématiquement « MeyerDoc », mais la plupart du temps sans indiquer la date et le lieu des documents et sans entrer dans la réflexion définitoire des lexèmes épineux. Les études grapho-phonétiques sont rares (cf. Schroeder 1932 et le travail décevant de Hug-Mander 1989, cf. Glessgen 1997), les travaux sur la morphologie ou la syntaxe inexistants.

Mais Paul Meyer a eu le grand mérite d'avoir ouvert la voie, conformément à son propos («L'important est de bien commencer», p. I), et c'est donc moins par l'apport empirique concret que par son apprentissage méthodologique qu'il a eu un impact sur la recherche future. 


\subsection{Clovis Brunel}

La réalisation de Clovis Brunel (1884-1971) a connu comme celle de Paul Meyer une phase préliminaire, représentée par la publication des documents du Gévaudan (Brunel 1916) et par un large tour d'horizon des plus anciens documents occitans (Brunel 1922). Ce dernier article profite pleinement des acquis de son maître et prédécesseur dans la chaire de philologie romane à l'École des Chartes. D'emblée, Clovis Brunel se concentre sur le seul domaine d'oc, en laissant de côté le francoprovençal : ce choix reste implicite, mais il est clair. Ensuite, il constate que la densité de la documentation ne permet pas de continuer dans la stricte lignée de Paul Meyer pour l'ensemble du territoire d'oc :

«Les facilités offertes par le groupement des premiers départements dont le nom commence par A (Hautes-Alpes, Basses-Alpes, Alpes-Maritimes), aussi bien que par la pauvreté des archives de ces pays de montagne, sont loin de se retrouver dans la suite de l'entreprise »(Brunel 1926, VII).

Il choisit par conséquent de réunir les plus anciens "textes d'ordre judiciaire et administratif ». Ce choix était à la fois plus systématique et plus réaliste que celui de Paul Meyer. Il se justifie également par la force d'inertie des genres textuels qui tendent à reproduire pendant des siècles des modèles une fois établis (cf. supra 2). Le terminus ante quem retenu (ca 1200) permettait à Clovis Brunel d'achever de son vivant son projet en deux volumes réunissant 541 actes; lors de la parution du Supplément, il avait toutefois, comme Paul Meyer auparavant, un âge avancé.

Par d'autres paramètres encore, Clovis Brunel a augmenté le côté systématique des DocLing :

- la présentation des éditions suivant un ordre chronologique, sans considération du lieu d'origine, ce qui facilite l'utilisation ;

- la tentative de datation et de localisation des documents à partir d'indices paléographiques et linguistiques ;

- la présentation homogène des éditions, avec un bref regeste et une partie bibliographique ;

- des critères d'éditions plus diplomatiques (indication des résolutions d'abréviation en italique, indication des lignes de l'original, indication des parties en latin, toujours au moyen de l'italique ; dans le deuxième volume, indication des séparations de mots);

- $\quad$ un glossaire synthétique [1926, 448-495 ; 1952, 228-258] et une table de noms propres [1926, 345-448 ; 1952, 179-227], plus développés que celui de Paul Meyer ;

- $\quad$ un relevé systématique de la morphologie nominale, pronominale et verbale [1926, XIII-LV ; 1952, XIII-XXXVI].

La description des caractéristiques des deux volumes permet de délimiter leur portée sous les différents aspects du temps, de l'espace, des genres textuels et, plus généralement, de la mise à l'écrit : 
(1) La couverture chronologique :

Les documents de Brunel se placent pour la quasi-totalité entre 1100 et 1200 (cf. la table chronologique 1952, 216-271) ; seulement six actes datent du XIe siècle, mais ce sont des textes mixtes (latin-occitan, voire latin-catalan).

Les documents édités rendent bien compte de l'état de production et de transmission des documents anciens originaux; Clovis Brunel a pu se valoir des notes de P. Meyer et du soutien de nombreux conservateurs d'archives pour ses relevés (cf. les remarques et remerciements dans Brunel 1922, 335 ; 1952, V, n. 2). Il reste, bien entendu, certaines lacunes, comme par exemple :

- le cartulaire de la Selve, publié par P. Ourliac et A.-M. Magnou en 1985, qui comporte près de 80 actes originaux antérieurs à 1201 (ainsi que 176 actes contemporains transmis par un cartulaire du début du XIII ${ }^{\mathrm{e}}$ siècle ; cf. Ourliac/Magnou 1985, 5-7);

- un nombre assez conséquent de textes mixtes des XI ${ }^{\mathrm{e}}$ et XII ${ }^{\mathrm{e}}$ siècles qui contiennent des renseignements précieux pour la mise à l'écrit de l'occitan (cf. le relevé détaillé de Belmon/ Vielliard 1997, 178-183);

- pour la toute première époque (antérieure à 1121), le corpus de l'ARTEM comporte six actes absents chez Brunel contre 24 actes publiés par lui (cf. Carles s.p. chap. 1.2.3).

On peut supposer de manière approximative que les deux volumes de C. Brunel réunissent pour l'époque concernée environ deux tiers des actes originaux conservés aujourd'hui.

(2) La couverture géographique :

La distribution géographique des documents occitans est très déséquilibrée, comme c'est le cas pour toutes les langues romanes médiévales. L'écrit et, à plus forte raison, l'écrit vernaculaire est tributaire des lieux d'écriture, à leur tour liés aux évolutions économiques et socio-politiques. La concentration sur le premier siècle de l'écrit vernaculaire réduit pratiquement la portée géolinguistique des volumes de Brunel au Languedoc et au Rouergue :

«nous avons un nombre prépondérant de documents pour une région qui peut être circonscrite entre les villes de Toulouse, Moissac, Villefranche-de-Rouergue, Rodez, Millau et Castres» (Brunel 1926, IX).

En tout, les deux volumes comportent vingt documents du Limousin, du Périgord, de l'Auvergne et du Vivarais, dix documents de la Provence et du Dauphiné ainsi que onze documents de la Gascogne (cf. Brunel 1926, LVI-LXIII ; 1952, XXXVII-XXXIX). Seulement 41 des 541 actes ne sont pas languedociens ou rouergats (7,5\%). L’idée de départ de Paul Meyer de couvrir le territoire méridional n'est donc aucunement réalisée par cette collection, malgré son indéniable atout de répondre à un objectif structurel clair et significatif. 
(3) Les genres textuels

Les genres textuels à l'intérieur de l'écrit documentaire sont également plus restreints que chez Paul Meyer qui a pu prendre en considération la diversification de l'écrit du XIII $/ \mathrm{XV}^{\mathrm{e}}$ siècle. Clovis Brunel souligne, certes, la richesse de son corpus :

«Les objets des textes sont assez divers. Une petite part comprend des documents d'économie domaniale, des notices, intitulées breve ou breu, de revenus dont il importe de rappeler l'assiette et le montant avec la sûreté de l'écriture. Pour le plus grand nombre, ont été trouvées des pièces, désignées par le mot carta, qui rapportent des faits juridiques. Si nous n'avons que deux rédactions de coutumes, nous sommes richement pourvus d'actes de la pratique. Parmi les chartes relatives aux personnes, nous comptons une série de serments de fidélité antérieurs à 1180, un aveu de vassalité, une réception d'office et surtout des professions d'oblat. La prépondérance appartient aux actes relatifs aux biens : inféodations et baux, lods, saisines ou déguerpissements, reconnaissances ou abandons de droits, cautions, impignorations ou dégagements, partages, dotations, legs, donations, donations rémunérées qui semblent des ventes déguisées, ventes déclarées et quittances. Les contestations ont donné lieu à des plaintes, enquêtes et attestations, rarement à des jugements, ce sont des accords amiables qui les préviennent ou terminent d'habitude » [1952, VIIs. et cf. encore la table chronologique citée].

Mais les chartes sont malgré tout largement dominantes dans les deux volumes. Il est certain que c'est un des genres textuels les plus complexes et les plus riches de l'écrit documentaire, mais d'autres genres fournissent d'autres informations, comme les simples inventaires. Ainsi, Clovis Brunel a exclu d'emblée une longue liste de redevances de la commanderie de Manosque, pourtant de la fin du XIIe siècle (1922, 338), puisqu'il n'a pas vu l'apport capital des noms de lieux et de personnes pour la linguistique historique.

\section{(4) La transmission des documents}

Clovis Brunel a choisi, de manière très rigoureuse, de renoncer à la prise en considération des copies de documents, même «transmises par des scribes du pays des actes originaux et à peu près du même temps » :

«Ont été seules retenues les pièces conservées en original, tant par souci de ne produire que des textes à l'abri de tout soupçon d'altération due à un copiste que par désir de ne pas donner à la publication une étendue trop grande» (1926, VIIs.).

Si le souci de la quantité est parfaitement compréhensible, la distinction déontologique entre originaux et copies contemporaines n'est pas aussi simple que Clovis Brunel la présente. Lui-même revient partiellement sur sa position dans le volume de supplément :

« Ont été jointes [...] plusieurs dizaines de documents [...] qu'un nouvel examen de leur caractère diplomatique nous fait classer aujourd'hui parmi les originaux plutôt que parmi les copies » (1952, V). 
La différence entre original et copie est, en effet, graduelle, surtout dans le domaine de l'écrit documentaire. Très souvent, les actes ont été produits en deux exemplaires pour les deux parties, parfois sous forme de chirographes, comme cela a été le cas habituel à Douai au XIII ${ }^{\mathrm{e}}$ s. (cf. Brunner 2014). Un acte connaît parfois une version préliminaire, comme il peut avoir été immédiatement transcrit dans un cartulaire etc. Leur valeur en tant que témoins pour la langue de l'époque demeure entière. Un original est certes toujours préférable à une copie, mais renoncer à l'étude des copies est une grave erreur qui rendrait d'ailleurs impossible le traitement des genres littéraires médiévaux.

Dans le cas concret de l'écrit occitan antérieur à 1200, la prise en considération des copies augmenterait le volume textuel disponible sans doute par un facteur deux ou trois. Mais, surtout, elle fournirait une vision géo-chronologique plus équilibrée de l'émergence de la scripturalité en langue d'oc puisque les textes les plus précoces ne sont souvent transmis que sous forme copiée (cf. par ex. pour l'Auvergne Chambon/ Olivier 2000, 107s.).

En conclusion, la décision double de clore la documentation en 1200 et d'exclure les copies conduit à renoncer à l'objectif d'une couverture équilibrée de l'espace géolinguistique. En même temps, cette décision a permis à Clovis Brunel d'achever un travail avec des dimensions bien déterminées et dans une logique structurée et cohérente. Les différences dans les approches entre Paul Meyer et Clovis Brunel permettent ainsi de mieux saisir les paramètres à prendre en considération dans le travail philologique appliqué aux textes documentaires.

Les travaux de Clovis Brunel ont connu une plus grande fortune que ceux de Paul Meyer notamment grâce aux études grapho-phonétiques et morphologiques d’Åke Grafström sur les chartes languedociennes (1958 et 1968), à leur utilisation intense par le FEW et, plus généralement, par la lexicographie de l'ancien occitan.

Notons toutefois que les études de Grafström ne reposent que sur 141 des 541 chartes de C. Brunel et que le FEW reste presque aussi sybillin dans la citation des volumes que pour celui de P. Meyer. Cf. par ailleurs pour le domaine graphophonétique les travaux de Kalman (1974) [chartes rouergates], Grafström (1978) [chartes limousines], Dinguirard (1979) [chartes gasconnes] et Soutou (1976) [Remarques sur une charte rouergate de 1161]. Quant au lexique, Max Pfister indique dans ses travaux sur l'occitan chaque fois la localisation et la datation des formes éditées ainsi que le passage dans les chartes, ce qui donne une idée plus claire de leur apport en termes de géolinguistique et de diachronie; cf. par ex. les attestations pour l'expression verbale « arouerg. jurar manbes 'jurer en personne, effectivement' (1182, Brunel 196,7), aalb. jurar marves (Vaour 1176-1199, p. 18, 20, 28, 101), arouerg. id. (1191-1195, Brunel 287, 6 ; 288, 28 ; BrunelS 507, 17 ; 515, 9) » etc. (Pfister 1970, 550).

S’ajoutent quelques rares études sur d'autres thématiques comme, notamment, celle de Linder (1970) sur quelques interrogations syntaxiques ou celles de Chambon $(1980$; 1987) sur l'identification - et l'édition - des formes toponymiques. 
Étant donné la grande richesse lexicale, onomastique, morphologique et syntaxique des chartes, il reste un potentiel important pour de futures études autant ponctuelles que monographiques sur ces deux volumes, tout comme pour celui de Paul Meyer. Mais, plus que ce dernier, Les plus anciennes chartes en langue provençale ont su s'imposer comme texte de référence et elles sont utilisées dans la mesure des recherches actuelles sur l'ancien occitan (cf. Chambon 2012).

En revanche, le souhait de Paul Meyer et de Clovis Brunel, d'avoir des continuateurs, n’a pas été exhaucé, puisque les prochaines étapes des DocLing ont privilégié le français au détriment de l'occitan.

\subsection{Jacques Monfrin}

Le projet des DocLing changea radicalement avec Jacques Monfrin (1924-1998) qui succéda en 1958 à son maître Robert Bossuat (ce dernier avait occupé pendant trois ans la chaire de linguistique romane de l'École des Chartes après deux décennies d'enseignement sur la chaire de sources narratives et littéraires). Bien qu'occitaniste, J. Monfrin décida de ne pas poursuivre le travail sur les documents occitans, mais d'ouvrir le chantier des documents d'oil et de reprendre celui des documents conservés en domaine francoprovençal. Par ailleurs, il impliqua bien plus que ses prédécesseurs d'autres collègues et de nombreux élèves dans le projet, ce qui permit un élargissement considérable de la documentation traitée. Grâce à ces divers soutiens, il put également procéder à un recensement bien plus systématique des régions concernées, toujours en partant de l'unité de base du département, conformément à l'organisation archivistique de la France. J. Monfrin établit également des critères d'édition plus rigoureux et cohérents et augmenta encore le soin de détail des volumes publiés (édition, notes, glossaire, index onomastiques). Enfin, les recensements se sont accompagnés dès lors d'une saisie photographique des documents édités.

Le résultat le plus visible de 'l'époque Monfrin' sont les sept volumes de textes documentaires publiés sous sa direction. Il s'agit, plus précisément, de trois volumes codirigés avec Lucie Fossier, comportant les plus anciennes chartes françaises de cinq départements contigus à l'est de Paris (Seine-et-Marne, Yonne, Aube, Haute-Marne et Vosges : 1974, 1975 et 1988), de deux volumes réunissant pour la Belgique les plus anciennes chartes françaises du Hainaut et de la Flandre $(1984$; 1987) et de deux volumes concernant le territoire francoprovençal, projetés par Mgr Pierre Gardette et codirigés avec lui $(1974 ; 1975)$ :

1974 : DocHM = Chartes en langue française antérieures à 1271 conservées dans le département de la Haute-Marne, ed. J.-G. Gigot [276 chartes]

1974 : DocFor = Documents linguistiques du Forez (1260-1498), ed. M. Gonon [pas de glossaire] [62 documents de nature variée, souvent longs]

1975 : DocV = Chartes en langue française [...] des Vosges, ed. J. Lanher, 1975 [135 chartes] 
1975 : DocLyo = Documents linguistiques du Lyonnais (1225-1425), ed. P. Durdilly [62 documents de nature variée, souvent très longs]

1984 : DocHain $=$ Chartes en langue française antérieures à 1271 conservées dans la province de Hainaut, ed. P. Ruelle [133 chartes]

1987 : DocFl $=$ Chartes en langue française [...] en Flandre orientale et Flandre occidentale, ed. R. Mantou [83 chartes]

1988 : DocAub = Chartes en langue française [...] de l'Aube, de la Seine-et-Marne et de l'Yonne, ed. D. Coq [103 chartes]

À ces volumes s’ajoutent deux publications antérieures, de qualité très différente :

DocPoit = La Du, Milan S., Chartes et documents poitevins du XIII siècle en langue vulgaire, 2 vol., Poitiers, 1960/1964 [concerne les dép. Vendée, Deux-Sèvres, Vienne, Charente-Maritime, Charente, comporte des documents antérieurs à 1300 ; ca 100 actes]

DocOis = Les plus anciennes chartes en langue française, t. $1:$ Problèmes généraux et recueil des pièces originales conservées aux Archives de l’Oise (1241-1286), ed. L. Carolus-Barré, 1964 [202 chartes]

Si l'édition de La Du intègre de nombreuses copies postérieures et s'avère très peu fiable d'un point de vue paléographique et philologique, l'ouvrage de Louis CarolusBarré avait, quant à lui, pleinement préparé la voie au projet poursuivi ensuite par Monfrin : «L'originalité et la qualité exceptionnelle de l'ouvrage ont fait que Jacques Monfrin l'a pris comme modèle pour les Documents linguistiques de la France » (Grübl 2013, 30).

Mais les volumes publiés ne donnent qu'une idée partielle de l'avancement du projet des DocLing. En effet, J. Monfrin avait suivi plusieurs thèses, surtout de l'École des chartes, s'inscrivant précisément dans l'idée initiale de Carolus-Barré. Entre 1964 et 1994 virent ainsi le jour sept ensembles de qualité, à l'état manuscrit, revus pour l'essentiel par J. Monfrin et presque tous annoncés par lui dès 1974 (XLIX) :

DocAisne_ms = Chartes de l'Aisne (1214-1350), ed. F. Grégoire-Ollivier, thèse de l'École des Chartes, 1964 [196 chartes]

DocCOr_ms = Chartes de la Côte d'Or (1239-1270), ed. V. Neveu, thèse de l'École des Chartes, 1988 [270 chartes, seule publication non annoncée par J. Monfrin en 1974]

DocDo_ms = Chartes de Douai (1204-1270), ed. M. Mestayer, ca 1974 [500 chartes]

DocDoub_ms = Chartes du Doubs (1233-1261), ed. M. Lefèvre, thèse de l'École des Chartes, 1975 / Id. (1260-1271), ed. J. Ducourtieux, thèse de l'École des Chartes, 1994 [en tout ca 250 chartes] DocMM_ms = Chartes de la Meurthe-et-Moselle (1232-1265), ed. M. Arnod, thèse de $3^{\mathrm{e}}$ cycle, 1974 [290 chartes]

DocPC_ms = Chartes du Pas-de-Calais, ed. P. Bougard, ca 1980 [128 chartes]

DocSom_ms = Chartes de la Somme, ed. J. Estienne, ca 1980 [ca 100 chartes]

L'héritage de J. Monfrin, transmis après sa disparition à son élève et successeur Françoise Vielliard, comportait par ailleurs de nombreuses photographies (noir et blanc) et certains sondages sur d'autres départements. 
Considérons synthétiquement les paramètres retenus pour Clovis Brunel.

\section{(1) La couverture chronologique}

Pour la série française de France et de Belgique, les DocLing couvrent intégralement les débuts de l'écrit documentaire vernaculaire jusqu'en 1270 environ. Le terminus ad quem garde une certaine souplesse, mais se place clairement avant la fin du XIII ${ }^{\mathrm{e}}$ siècle quand le volume textuel commence à augmenter considérablement. C'est donc un choix semblable à celui de Clovis Brunel.

Les documents provenant du territoire francoprovençal suivent en revanche la logique des documents de Paul Meyer. Ils couvrent essentiellement le XIV ${ }^{\mathrm{e}}$ ainsi qu'une partie du XV $\mathrm{XV}^{\mathrm{e}}$ siècle car les sources vernaculaires sont encore rares au XIII ${ }^{\mathrm{e}}$. Les volumes donnent un recensement intégral pour les régions à l'étude et éditent ensuite un choix de documents.

(2) La couverture géographique

Les volumes publiés et manuscrits réunissent des documents de la majeure partie des départements du nord-est du territoire d'oïl qui sont, avec l'Angleterre, les plus précoces dans la production textuelle. En revanche, les régions à l'ouest de la Picardie, de la Champagne et de la Bourgogne sont restées en suspens, de même que Paris et ses environs immédiats. Curieusement, le sondage effectué dans les Archives Nationales et dans la Bibliothèque Nationale n'a pas été poursuivi (Monfrin 1968, 32 indique près de 500 pièces françaises avant 1271), alors que ces deux lieux de conservation sont les plus facilement consultables par les élèves et professeurs parisiens.

Cette couverture reste toutefois virtuelle puisque les volumes manuscrits ne sont pas accessibles aux chercheurs. Pour le domaine d'oïl en France, seuls les quatre volumes des DocOis, DocAub, DocHM et DocV sont disponibles, ce qui donne une idée géolinguistique encore très insuffisante.

(3) Genres textuels, transmission des documents, langues

J. Monfrin considère avec plus de prudence encore les questions de transmission :

« [...] la différence entre les originaux et les copies est moins de nature que de degré. Les premiers autant que les secondes peuvent être trompeurs, et il convient, avant de les interroger, de soigneusement vérifier leur état civil » (Monfrin 1968, 46).

Les DocLing incluent ainsi régulièrement des copies contemporaines, même s’ils restent ciblés sur les originaux.

Quant aux genres textuels, les séries françaises contiennent dans l'immense majorité des chartes, alors que la série francoprovençale en est presque exempte : ici dominent les documents de gestion et diverses listes.

D'un point de vue linguistique, les 62 documents du Forez correspondent comme ceux de l'Ain pour la plupart à une scripta de type oïlique, mais les 5 documents considérés par les éditeurs à juste titre comme véritablement francoprovençaux 
( $\left.\mathrm{n}^{\circ} 4-7,14\right)$ sont pour certains assez volumineux (le Terrier de Chazelles 1290 et les Comptes de l'hôtel du comte de Forez 1322/1323). L'important volume sur le Lyonnais (près de 600 pages) présente en revanche une scripta essentiellement francoprovençale, ce qui souligne le rôle de Lyon comme épicentre de cette langue.

(4) Utilité et utilisation

Dans cette troisième phase du projet, les avancées des DocLing sont considérables concernant l'avancement de l'édition : grâce aux volumes publiés sous la direction de Jacques Monfrin, Lucie Fossier et Pierre Gardette ainsi que le volume de Louis Carolus-Barré, on disposait à présent de près de 800 chartes du XIII ${ }^{\mathrm{e}}$ siècle et d'une remarquable série de documents francoprovençaux du XIV siècle, tous dans un état éditorial exemplaire. S’ajoutait l'édition manuscrite de plus de 1700 chartes oïliques, dans un état encore préliminaire mais très avancé.

Les éléments analytiques en revanche ne sont pas développés. Les volumes de la série française disposent chacun d'un glossaire soigné qui suit la conception établie par Clovis Brunel. Ces glossaires ont une réelle utilité et ont pu être exploités par la lexicographie de l'ancien français, notamment par le DEAF. Leur utilisation en lexicologie est plus circonscrite (cf. par ex. Drüppel 1984), parce que les textes documentaires sont généralement peu utilisés, que l'éclatement des glossaires dans six volumes distincts ne facilite pas le travail et, enfin, parce que la glossographie traditionnelle des langues gallo-romanes médiévales ne répond que de manière très insatisfaisante aux attentes de la lexicologie actuelle qui a fait siens les acquis méthodologiques de la lexicographie monolingue. Quant à ce dernier point, Jean-Pierre Chambon a montré le potentiel lexicologique qui réside dans les DocLing, en proposant une réécriture de l'entrée don(s) du volume DocHM :

dons 13, 4, 14, 7, 78, 11, suj. sing., don $4,6, \mathbf{6}, 5, \mathbf{3 0}, 2, \mathbf{5 6}, 3, \mathbf{6 7}, 7$, $\mathbf{8 8}, 3, \mathbf{8 9}, 5, \mathbf{9 3}, 8, \mathbf{9 4}, 8, \mathbf{1 0 5}, 3$, $\mathbf{1 1 0}, 6, \mathbf{1 1 7}, \iota_{4}, \mathbf{1 1 9}, 6, \mathbf{1 3 9}, 8$, 161, $12, \mathbf{1 6 7}, 7, \mathbf{1 7 0}, 2, \mathbf{1 7 4}, 3$, 184, 10, 186, 4, 189, 7, 204, 10, 215, $10, \mathbf{2 2 9}, 8, \mathbf{2 4 8}, 5, \mathbf{2 5 5}, 6$, ^1 b, 24, dom 7, 7, 201, 15, doin 68, 5, 172, 5, rég. sing.; dons 133, 22, 133 a, 22, `3, 5, rég. plur., don. - Dons 164, 10, 239, 4, 240, 4, 241, 4, suj. sing., don 22, 19, 238, 3, 242, 3, 242 a, 5, droit de donner.

(DocHM 1974, 466) 
DoN s. m. I (partout sauf dom 7,7, 201,15; doin $68,5,172,5$ ).

1. [Souvent en liaison syntagmatique avec aumone ou var., rarement avec aquitance, otroi ou quitemant] "action d'abandonner gratuitement à qn la propriété de qch" (1234-1269 ou 1270) 4,6, 6,5 (et cest don et ceste aumone ont il donee et otroié par lou louz et par l-otroi de mon signor Orri de Mairé), 7,7, 13,4, 14,7, 30,2, 56,3, $68,5,78,11,89,5,110,6,117,4,119,6,133,22,133 \mathrm{a}, 22,139,8,167,7,170,2,172,5,184,10$, 189,7 (par opp. à vandue s. f.), 201,15 (par opp. à vandue), 204,10, 215,10 (par opp. à eschange), 229,8, 248,5, 255,6.

- Sg. à valeur coll. (trad. mil. $13^{\mathrm{e}} \mathrm{s}$.) $* 1 \mathrm{~b}, 24$.

- Loc. verb.

- faire don de "abandonner gratuitement (à qn) la propriété de (qch)" (1258/9) 93,8-9, 94,8;

- [Fig. étymol. ayant une valeur de renforcement] doner par don "abandonner (à qn) la propriété de (qch), de manière purement gratuite" (1259-1265), 88,3, 105,3, 174,3, 186,4; doner pour don "id." (1264) 170,2.

2. Par méton. "ce qu'on a abandonné gratuitement à qn (sur qch)" (1263) 161,12 ([un abbé et un prieur] demandent raison en la proprieté dui don de l-iglise).

3. Par restr. [souvent en liaison syntagmatique avec collation/-m ou presentacion] "droit de faire un don (d'un revenu ecclésiastique)" (1249-1269) 22,19 (de cele dite chapelerie je deteng lo don tant comme je viverai), 164,10, 238,3, 239,4,5, 240,4, 241,4, $242,3,242 \mathrm{a}, 5$.

Bibl. - GdfC (ne distingue pas 1 et $2 ; \emptyset 3$ ); $T L$ (ne distingue pas 1 et $2 ; \emptyset 3$ ); FEW 4, 138b, DONUM (1 non défini; $\emptyset$ 2, 3); TLF (1 depuis Passion; 2 depuis Roland; Ø 3).

(Chambon 2006, 136s. ; cf. le commentaire détaillé ib. 130-138)

Pour la série francoprovençale, Mgr Gardette avait envisagé un glossaire unique pour les trois volumes projetés. Ce choix était très judicieux, mais il a eu comme triste conséquence que les deux seuls volumes publiés ne sont accompagnés ni de glossaire ni d'index de noms propres, ce qui a fortement nui à l'utilisation de ces précieuses éditions.

En scriptologie, Anthonij Dees s'est basé pour son Atlas [...] des chartes françaises sur les trois volumes des DocOis, DocHM et DocV (cf. la liste de la 'provenance des chartes', Dees 1980, 307-312) ; malheureusement, la juxtaposition de ces éditions fiables avec de nombreuses autres paléographiquement et philologiquement peu sûres (comme par exemple les DocPoit) réduit le rendement de l'analyse scriptologique.

En conclusion, avec Jacques Monfrin, les DocLing sont pleinement présents dans les trois territoires linguistiques de la Galloromania. La qualité et le soin des éditions font le grand atout de cette collection qui garde comme ultime objectif un recensement systématique de l'écrit documentaire médiéval. Pourtant, les réalisations accessibles de l'époque de Jacques Monfrin sont restées, plus encore que le volume de Paul Meyer, une œuvre inachevée, géographiquement disparate et difficile à exploiter. Quant aux archives léguées par J. Monfrin, elles formaient un héritage peu aisé à gérer (cf. infra 4.1). 
Dans un certain sens, le projet de Monfrin s'inscrit pleinement dans l'esprit d'une époque gaullienne, tout comme le Trésor de la langue française ou l'entreprise des Nouveaux atlas linguistiques de la France. Pour le TLF, grâce à des subventions considérables, il a été possible de saisir les données textuelles trois fois successives et d'achever sur cette base le 'Nouveau Littré' projeté ; pour les NALF, en revanche, de nombreux volumes sont restés, encore aujourd'hui à l'état de manuscrits, et peu de séries ont connu ne serait-ce que des index lexicologiques conséquents (comme par ex. Dondaine 2002). Le caractère monumental de l'entreprise a en quelque sorte empêché sa réalisation dans un temps défini.

S'ajoutent, dans le cas des DocLing, deux problèmes intrinsèques : tout comme les NALF, le projet de Monfrin est pensé dans une logique de répertoire plus que dans une logique d'utilisation; des éditions de qualité et des cartes d'atlas bien pensées sont naturellement d'excellentes sources pour la linguistique historique et variationnelle. Mais la mise à disposition des matériaux doit s'accompagner en même temps d'analyses linguistiques pour en susciter d'autres. Dans cette logique, le TLF est devenu un centre d'Analyses et traitements informatiques de la langue française, ce qui lui a garanti une place dans le paysage de la recherche actuelle. Le deuxième problème réside dans l'immensité de la matière à traiter que Jacques Monfrin a sousestimée, tout comme ses prédécesseurs et - il faut bien l'avouer - nous-mêmes, dans la $4^{\mathrm{e}}$ phase des DocLing.

\section{Les DocLing actuels}

\subsection{Les textes réunis actuellement dans l'édition électronique des DocLing}

Après la disparition de Jacques Monfrin en 1998, Françoise Vielliard, son successeur depuis 1992, hérita des matériaux des DocLing. Par pur hasard, nous nous sommes intéressé à ce projet centenaire endormi depuis près de deux décennies. Françoise Vielliard mit alors très généreusement à notre disposition ces matériaux, revus de près par elle, ainsi que son expérience et le soutien institutionnel de l'École des chartes.

Notre propre conception de la suite à donner au projet a connu d'importantes évolutions au cours des quinze dernières années : au début, nous souhaitions intégrer, à l'instar de Paul Meyer, les textes documentaires lorrains des XIV et XVe siècles dans un projet impliquant la philologie informatique (cf. Glessgen 2001a). Après avoir constaté, sur la base de quelques sondages, le caractère très traditionnel de ces genres textuels (cf. Glessgen 2004), nous nous sommes convaincu qu'il était indispensable de documenter avant tout les débuts de l'écrit vernaculaire, précisément dans la logique de Clovis Brunel et de Jacques Monfrin. L'aide amicale de Françoise Vielliard et d'Olivier Guyotjeannin ouvrait cette voie qui nous mena aussi à renoncer dans un premier temps à l'élargissement géo-chronologique de la série occitane. Nous nous 
sommes donné l'objectif d'avancer voire de clore le dessein de Jacques Monfrin sous les auspices de la philologie informatique : informatiser les volumes existants, saisir et corriger les volumes manuscrits, élargir géographiquement les recensements, en nous concentrant sur la (grande) moitié orientale du territoire d'oïl et en excluant les régions situées à l'ouest de la Picardie, de Paris et de la Bourgogne. Ce choix était dicté autant par notre intérêt tout personnel pour la Lorraine que par la distribution géographique des matériaux disponibles. En même temps, cette orientation rend compte des épicentres de l'écrit documentaire oïlique et permet par ailleurs de faire la transition avec le domaine francoprovençal.

Si notre choix nous semble toujours rationnel, il a largement sous-estimé la complexité de la matière. Autant la saisie et la révision philologique des volumes non publiés que le développement de l'environnement informatique du projet se sont avérées extrêmement lourds, au-delà de toute attente.

Les efforts conjoints et l'enthousiasme de très nombreux collègues et amis, élèves et jeunes chercheurs (cf. <www.rose.uzh.ch/docling>), de même que le soutien financier considérable de plusieurs institutions académiques (cf. ib.) ont toutefois permis de réunir en 2014 les onze séries suivantes sous un format électronique homogène prévoyant des interrogations linguistiques poussées (cf. DocLing-2) :

\begin{tabular}{|c|c|c|c|c|c|}
\hline sigle & $\begin{array}{l}\text { département } \\
\text { (ou lieu } \\
\text { d’écriture) }\end{array}$ & dates extrêmes & $\begin{array}{l}\text { auteur de } \\
\text { l'édition papier }\end{array}$ & $\begin{array}{l}\text { auteur ou } \\
\text { responsable } \\
\text { de l'édition } \\
\text { électronique }\end{array}$ & $\begin{array}{l}\text { nombre } \\
\text { de doc. }\end{array}$ \\
\hline DocDo & Douai & $1204-1270$ & Mestayer ca 1970 & Brunner 2014 & 506 \\
\hline DocJu & Jura & $1243-1296$ & & Muller 2014 & 95 \\
\hline DocHM & Haute-Marne & $1232-1275$ & Gigot 1974 & Kihaï 2009 & 276 \\
\hline DocHS & Haute-Saône & $1242-1300$ & & Muller 2014 & 132 \\
\hline DocMa & Marne & $1234-1272$ & & Kihaï 2009 & 230 \\
\hline DocMe & Meuse & $1225-1270$ & & Matthey 2009 & 237 \\
\hline DocMM & $\begin{array}{l}\text { Meurthe-et- } \\
\text { Moselle }\end{array}$ & $1232-1265$ & Arnod 1974 & Glessgen 2014 & 290 \\
\hline DocNi & Nièvre & $1289-1330$ & & Alletsgruber 2014 & 34 \\
\hline DocR & $\begin{array}{l}\text { Chancellerie } \\
\text { royale }\end{array}$ & $1241-1300$ & & Videsott 2014 & $\begin{array}{l}140 \text { (dont } \\
20 \text { copies) }\end{array}$ \\
\hline DocSL & Saône-et-Loire & 1227-1331 & & Alletsgruber 2014 & 100 \\
\hline DocV & Vosges & $1235-1275$ & Lanher 1975 & Trotter 2014 & $146 ?$ \\
\hline
\end{tabular}

Cette collection comprend donc deux des volumes anciennement publiés (DocHM et DocV), deux des volumes anciennement manuscrits (DocDo et DocMM qui ont fait l'objet d'une révision intégrale) ainsi que plusieurs nouvelles séries élaborées par quatre de nos élèves ainsi que par notre ami Paul Videsott. L'édition électronique 
comporte pour la plupart une reproduction photographique de ces 2185 documents. Pour documenter les débuts de l'écrit dans les différentes régions, nous avons reculé la date butoir jusqu'en 1300 voire, pour la Bourgogne, jusqu'en 1331. Les documents réunis couvrent une zone relativement continue du Nord et, surtout, du Nord-Est de la France (Flandre, Champagne, Lorraine, Franche-Comté, Bourgogne) et incluent également la chancellerie royale.

La réalisation actuellement disponible pourrait connaître dans les années à venir divers nouveaux apports, notamment ceux :

- des volumes publiés par Louis Carolus-Barré (saisis sous la direction de Benoît M. Tock et actuellement étudiés par Klaus Grübl) et par Dominique Coq,

- des volumes publiés des séries belge et francoprovençale ainsi que les documents récemment publiés du Luxembourg (Holtus/Overbeck/Völker 2003) et de la Suisse romande (DocJuBe, DocNeu),

- du volume de la Moselle, préparé par notre regrettée amie d'études Martina Pitz et en cours d'étude par Frédéric Duval, à son tour successeur de Françoise Vielliard depuis 2011,

- d'une série importante de textes de la Prévôté de Paris (1260-1300, ca 250 documents), en cours d'étude par Paul Videsott,

- d'une série anglo-normande, étudiée par David Trotter (cf. Trotter 2015, §4.3),

- enfin de certains des manuscrits restés en suspens (DocAisne, DocCOr, DocDoub, DocPC, DocSom).

La base textuelle constituée permettra facilement l'intégration de ces nouvelles séries, intégration qui suppose toutefois un travail éditorial considérable.

\subsection{Les caractéristiques philologiques et informatiques des DocLing}

Les principes de réalisation des DocLing sous leur forme actuelle sont, en principe, indépendants des genres textuels en question. La gestion informatique permet l'application de critères d'édition à la fois plus rigoureux et plus souples, par l'encodage neutre de type XML des textes. Les interrogations linguistiques prévues sont également pertinentes pour tout genre textuel. Cela vaut aussi pour la présentation parallèle de la transcription et des images.

Pour l'édition, la mise en ligne permet de distinguer trois vues différentes sur les mêmes textes :

- une vue diplomatique (organisée d'après les lignes du manuscrit et reproduisant les séparations des mots, la ponctuation et les majuscules de l'original, les abréviations étant résolues en italiques), comme par ex. :

1 Conue chose soit atoz que li abes et li chapitles de salinvas · at laissie a wirion

2 et huillon les dous freres de geverlise les anfanz bertran bacheler $\cdot$ XIII · jor

3 nas de terre treisse $\cdot$ en la fin de geverlise $\cdot$ et a lor oirs · parmi $\cdot$ XIII - deniers de cens $\cdot$ et

4 ·II $\cdot$ himas de blef $\cdot$ lun davoine $\cdot$ lautre de froment $\cdot$ et sil ne paievent a jor 
5 nomei a la feste sent remi a giverlise en la maison de salinvas · que lon se tan

6 roit a la terre $\cdot$ et ce que sus averoit. Si est ensi devisee. qau Tramble en

7 at $\cdot$ IIII $\cdot$ jornas $\cdot$ un par lui $\cdot$ et $\cdot$ III $\cdot$ ensemble $\cdot$ et en la voie $[. .$.

(DocMM ch. $2:$ 1234, AbbSalival)

Cette vue diplomatique peut être immédiatement comparée avec la reproduction photographique (en couleurs sur le site) :

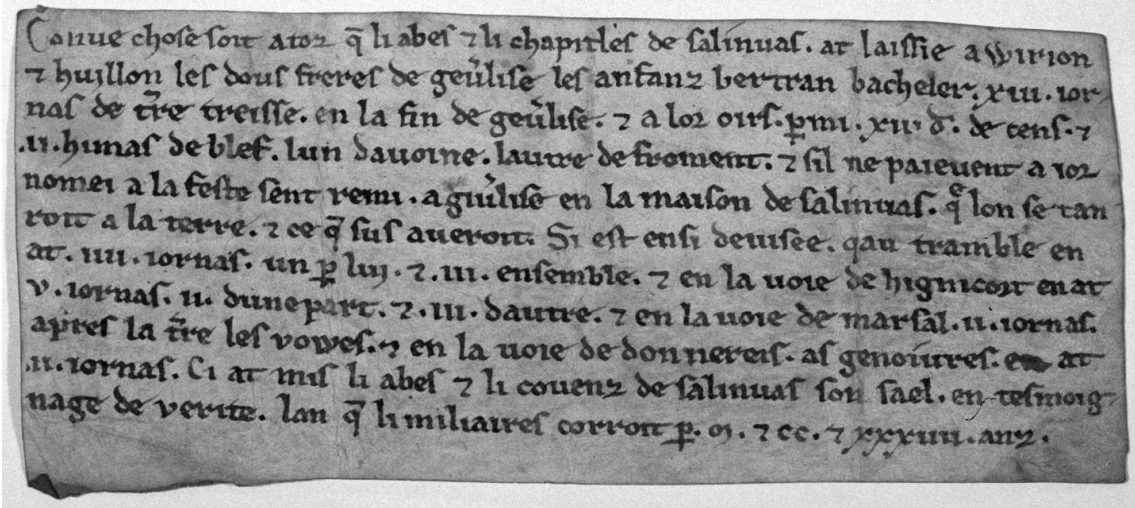

- une vue interprétative (qui introduit des séparations de mots, la ponctuation et les majuscules selon l'usage actuel, ajoute des accents, des numéros de structuration sémantico-formelle et, le cas échéant, des paragraphes) :

1 Conue chose soit à toz $\mathbf{2}$ que li abes et li chapitles de Salinvas at laissié à Wirion et Huillon, les dous freres de Geverlise, les anfanz Bertran Bacheler, 3 XIII jornas de terre treisse, en la fin de Geverlise, et à lor oirs, 4 parmi XIII deniers de cens et II himas de blef, l'un d'avoine, l'autre de froment ; 5 et s'il ne paievent à jor nomei à la feste sent Remi, à Giverlise en la maison de Salinvas, que l'on se tanroit à la terre et ce que sus averoit [...]

- une vue mixte (qui rend les séparations médiévales transparentes, combine la ponctuation médiévale [au milieu de la ligne] avec la moderne et les majuscules médiévales [en gras] avec les modernes). Cette vue mixte est également celle qui peut être exportée pour tous les documents sous un format pdf : 


\section{PUBLICATIO}

1 Conue chose soit à-toz

\section{EXPOSITIO}

2 que li abes et li chapitles de Salinvas-at laissié à Wirion $\backslash_{2}$ et Huillon, les dous freres de Geverlise, les anfanz Bertran Bacheler, 3 /.XIII/. jor $\backslash_{3}$ nas de terre treisse· en la fin de Geverlise· et à lor oirs· 4 parmi $\cdot \mathrm{XIII} /$. deniers de cens- et $\backslash_{4} \cdot \mathrm{II} /$. himas de blef l'un d' avoine l'autre de froment 5 et s'il ne paievent à jor $\backslash 5$ nomei à la feste sent Remi- à Giverlise, en la maison de Salinvas ${ }^{[i]}$. que l'on se $\tan \backslash 6$ roit à la terre- et ce que sus averoit. $6 \mathrm{Si}$ est ensi devisee- q'au

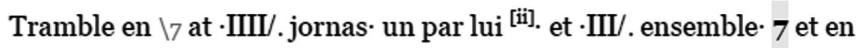
la voie de Hignicort en at $\backslash 8 \mathrm{~V} /$. jornas II/. d'une part et 'III/. d' autre $\mathbf{8}$ et en la voie de Marsal $\cdot \mathrm{II} /$. jornas $\backslash_{9}$ aprés la terre les Vowes $^{\text {[iii]. }} 9$ et en la voie de Donnereis- as Genoivres- en at $\backslash 10 \cdot \mathrm{II} /$. jornas.

La base de données actuelle de type MySql repose sur une organisation sous forme non pas textuelle, mais de listes; les textes de départ sont donc éclatés lors de l'intégration dans la base et reconstitués lors de la lecture, ce qui permet une gestion plus rapide et plus cohérente des interrogations et informations linguistiques. La part de programmation dans le projet actuel a occupé une place prépondérante. La philologie informatique ouvre, certes, des voies nouvelles, mais elle comporte de grandes pesanteurs. Qui plus est, elle est difficile à financer puisque les institutions d'aujourd'hui considèrent à tort qu'il s'agit là d'une part préliminaire et non-scientifique du travail. Grâce à l'engagement partiellement bénévole de plusieurs collaborateurs et amis, la programmation a pu être achevée, sous une architecture à la fois claire et fonctionnelle, en 2014, après plus d'une décennie (cf. Glessgen 2011b). Elle a la qualité d'être utilisable par tout projet philologique d'une certaine complexité, même s'il faut prévoir, là encore, un budget circonscrit pour son adaptation à d'autres ensembles textuels.

Malgré ces lourdeurs, la mise en ligne des documents s'avère le vecteur essentiel pour dépasser l'éclatement de l'information linguistique qui est intrinsèque aux DocLing depuis ses débuts: dans l'édition de Paul Meyer, tout lexème doit être cherché en quatre glossaires différents, et l'on doit se reporter aux références indiquées pour chaque occurrence afin d'identifier le lieu et la date du texte; la même procédure s'impose pour les deux glossaires de Clovis Brunel ou les six glossaires de l'époque Monfrin. Dans l'édition électronique actuelle, la recherche d'une forme 
apporte immédiatement l'ensemble des occurrences avec l'indication de la date, de la provenance, du contexte et, si possible, du rédacteur. Pour les recherches graphématiques ou morphologiques, la gestion parallèle des 2.000 chartes ouvre les mêmes perspectives permettant, enfin, de considérer cet ensemble documentaire comme une unité et un corpus de référence avec, actuellement, près de 900.000 occurrences.

\subsection{Les interrogations linguistiques}

La quasi-totalité des documents réunis actuellement sont des chartes, à côté de quelques rares testaments et une charte-loi. Le genre des chartes est toutefois d'une richesse notable, autant syntaxique qu'onomastique. Les plus anciens actes permettent notamment de cerner l'élaboration d'une syntaxe complexe qui commence à se détacher de l'oralité dialogale et narrative sous-jacente. Les textes sont datés avec précision et il est également possible d'identifier pour la plupart d'entre eux le lieu d'écriture responsable pour leur rédaction (cf. Glessgen 2008 et déjà Carolus-Barré 1964, LXXXIII-CVII, cf. Grübl 2013, 32s.). Nous disposons donc d'un ancrage diasystématique sûr, qui fait la qualité particulière de l'écrit documentaire.

Le logiciel permet des interrogations à partir des mots graphiques et à l'aide des 'expressions régulières' (= des chaînes de caractères suivant une syntaxe précise), par ex. :

- $\quad$ "^contre. ${ }^{\star "}$ recherche toutes les formes qui commencent par contre- [donc contredire etc., contredisor, contrefaire etc., contreforchier, contremont, contreseel, contreval, contrewage]

- $\quad$ "[b|u]les ?\$", toutes les formes qui se terminent en -ule(s) ou -ble(s) [donc toutes les formes qui correspondent aux variantes suffixales -a(i)ble, -auble et -aule]

Celxa permet toutes sortes d'interrogations graphématiques ou lexicologiques dont les résultats peuvent être exportés par la suite dans un document Excel ou Word. Nos analyses grapho-phonétiques restent encore partielles, mais il est dès à présent patent, que les DocLing fournissent une nouvelle référence pour la description du paysage scriptologique de l'ancien français (cf. Videsott 2013 ; Grübl 2014).

Quant au lexique, la lemmatisation des DocLing est en cours, et toute forme lemmatisée est intégrée par un processus semi-automatique dans la version électronique du DEAF (DEAF-pré). Dans un premier temps, les formes des DocLing sont simplement répertoriées sous les lemmes du DEAF, avec leurs dates et contextes (permettant de voir à tout moment le contexte complet par un hyperlien avec la base des DocLing) ; dans un deuxième temps, il sera possible de les traiter à l'intérieur de la base du DEAF autant du point de vue sémantique que syntagmatique (cf. Glessgen/ Tittel 2015). Par cette voie, l'éternelle scission entre la lexicographie d'une langue ancienne et sa glossographie - circonscrite à un ensemble de textes donnés - est dépassée, et il est possible de fonder une description lexicologique de la langue immédiatement sur des bases textuelles (cf. Glessgen 2007). 
Pour des analyses syntagmatiques et syntaxiques, les données de la base DocLing pourront être exportées, sans doute en 2016, pour être traitées par le TreeTagger et les outils syntaxiques développés par Achim Stein (cf. <www.uni-stuttgart.de/lingrom/ forschung/ressourcen>). L'identité de la structure des données et des balises morphologiques facilitent la programmation des interfaces nécessaires. Enfin, les données textuelles peuvent être exportées par les utilisateurs sous un format XML qui permet toute analyse souhaitée.

\section{Perspectives}

Les éditions de textes documentaires, surtout anciennes, sont nombreuses, même sans prendre en considération le latin médiéval. Jacques Monfrin dresse un aperçu impressionnant de ses précurseurs entre 1829 et 1964 (Monfrin 1974, XI-XXXIX ; cf. aussi la synthèse régionale des sources connues pour l'Auvergne et le Velay, Chambon/Olivier 2000, 105-110, 119-126 et, pour le Mauriacois et le Sanflorain, Olivier 2009, XXIX-XL). La place des DocLing ressort plus précisément du vol. 4 de l'Inventaire (InvSyst) des documents romans antérieurs à 1250, intégralement consacré aux chartes françaises et occitanes. Ce volume, réalisé par J. Hartmann, n'intègre pas tous les documents qui reposent dans les archives, mais il rend bien compte des documents publiés jusqu'en 1997. Les DocLing occupent une part réelle parmi ces éditions disponibles pour les XII et XIII ${ }^{\mathrm{e}}$ siècles, déjà par leur présence quantitative. Mais ils se détachent surtout par leur grande fiabilité philologique et éditoriale et la volonté d'une emprise systématique sur la documentation disponible.

Il n'y a pas de doute que le projet des DocLing a sa raison d'être et qu'il joue même un rôle paradigmatique pour les travaux en philologie linguistique. Les conditions de développement des DocLing sont également très favorables depuis les débuts parce que la gestion du patrimoine archivistique de la France est exemplaire et que les conservateurs tout comme les médiévistes historiens ou linguistes soutiennent pleinement ce projet.

Or, nous avons vu que malgré tout, la réalisation des DocLing n'a jamais été aisée et qu'elle est toujours restée (très) partielle. On peut légitimement se demander où est l'erreur. Le problème fondamental nous semble résider dans l'immensité de la matière à étudier. Aucun savant ni même aucun groupe de travail n'aurait eu l'idée farfelue de traiter dans le cadre d'un seul projet l'écrit littéraire, religieux ou médico-biologique gallo-roman - alors que c'est bien cela que les DocLing ont voulu entreprendre pour les textes documentaires, bien plus nombreux et dont le traitement philologique n'est pas plus facile. L'idée de départ des DocLing se trouve donc tout simplement en décalage maximal avec la réalité de la transmission textuelle. Cela ne veut pas dire qu'il faille renoncer à une approche structurée de ce patrimoine écrit, mais il faut prendre la mesure de ses dimensions déroutantes et cerner des ensembles plus circonscrits. 
Un deuxième problème annexe réside sans doute dans la concentration (trop) forte des DocLing sur l'édition en tant que telle. Pour rendre les chartes parlantes, il faut les placer dans leur contexte de genèse, il faut mener des analyses ciblées sur les différents domaines linguistiques pertinents, de la ponctuation (comme par ex. Mazziotta 2009 sur un corpus wallon) à la subordination (comme par ex. Gévaudan/ Glessgen s.p. sur les DocLing), et il faut les comparer avec d'autres genres textuels contemporains. Il nous semble même indispensable de traduire un certain nombre de documents en français moderne pour faciliter la compréhension de leur lexique particulier et de leur syntaxe ardue à des jeunes chercheurs qui n'ont aucun mal à se familiariser avec des textes littéraires médiévaux grâce à de nombreuses éditions bilingues. Ici, la recherche se heurte en outre aux aléas de la formation universitaire, trop peu diachronique et, en France, exclusivement ciblée sur les textes littéraires.

D’autres projets récents sont consacrés à l'écrit documentaire gallo-roman. Pour le latin médiéval, la collection des près de 5000 Chartes originales antérieures à 1121 conservés en France de l'ARTEM a été publiée, après quatre décennies de préparation, en 2010 ; elle est complétée par la collection des Chartae Galliae qui réunit près de 40 000 actes, pour la plupart copiés, antérieurs à 1300, en incluant également des textes vernaculaires (dont les volumes publiés des DocLing qui ont pu être saisis par cette voie, grâce à B. Tock). Pour le lexique, mais également pour la graphématique et, partiellement, la morphologie et la syntaxe des anciennes langues gallo-romanes, ce sont des sources précieuses.

Dans le domaine plus spécifique de l'occitan, la publication des registres de comptes des consuls de Clermont-Ferrand par Anthony Lodge fournit un apport considérable aux études régionales (Lodge 2006 ; 2010) ; en complément, le dictionnaire de Philippe Olivier de l'ancien auvergnat repose, à son tour, sur plus de 10000 pages transcrites préalablement dont les éléments lexicaux ont été extraits avec leur contexte (Olivier 2009). Ajoutons par ailleurs le projet de la Concordance de l'occitan médiéval (COM), initié par Peter Ricketts et continué par Dominique Billy dont la section en cours (COM-3) comporte également les textes documentaires.

Tous ces projets partagent avec les DocLing une attention philologique et une pensée systématique qui sont, toutes deux, indispensables pour approcher l'écrit documentaire. Les DocLing gallo-romans restent donc une mesure paradigmatique pour l'étude de ce patrimoine qui offre, parmi tous les genres textuels, les perspectives les plus prometteuses pour la recherche actuelle. L'histoire des DocLing montre très clairement l'importance de segmenter le domaine à l'étude de manière préliminaire, d'après des critères abstraits (le temps, l'espace, les langues, les genres étudiés) et selon l'état de transmission et d'édition des sources disponibles. Si dans le cas des textes littéraires, une telle segmentation est préfigurée par les sous-genres traditionnels, les auteurs et les textes, pour les textes documentaires, elle demande une réflexion ad hoc pour chaque étude nouvelle. Cette réflexion est en même temps indispensable puisque personne ne peut embrasser seul la matière. 


\section{Bibliographie}

Nous renonçons dans ce cadre à l'indication bibliographique des dictionnaires gallo-romans de référence (comme FEW ou DEAFpré) ; cf. le Complément du FEW ( $3^{e}$ édition Jean-Paul Chauveau et al., Strasbourg, 2010) et le Complément bibliographique du DEAF en ligne (<www.deaf-page. de>).

\section{1 Éditions de textes documentaires}

ARTEM = AA.VV. (2010), Chartes originales antérieures à 1121 conservés en France, publication en ligne (<www.cn-telma.fr/originaux>).

Brunel, Clovis (1916), Documents linguistiques du Gévaudan, Bibliothèque de l'École des chartes 77, 5-57, 241-285.

Brunel, Clovis (1926), Les plus anciennes chartes en langue provençale. Recueil des pièces originales antérieures au XIII' siècle. Publiées avec une étude morphologique, Paris, Picard.

Brunel, Clovis (1952), Les plus anciennes chartes en langue provençale. Recueil des pièces originales antérieures au XIII' siècle. Supplément, Paris, Picard.

Chartae Galliae = AA.VV. (2014), Chartae Galliae, publication en ligne (<www.cn-telma.fr/chartaegalliae>).

COM = Ricketts, Peter/Billy, Dominique, Concordance de l'occitan médiéval : COM 1 (troubadours) et COM 2 (textes narratifs), ed. Peter Ricketts, cd-rom, Brepols ; COM 3 (textes non-littéraires) en préparation, ed. Peter Ricketts/Dominique Billy.

DocAisne_ms = Françoise [Grégoire-]Ollivier, Documents linguistiques de la Picardie orientale (1214-1350), thèse de l'École des Chartes, 1965.

DocAub = Dominique Coq, Documents linguistiques de la France (série française, vol. 3), Chartes en langue française antérieures à 1271 conservées dans les départements de l'Aube, de la Seine-etMarne et de l'Yonne, Paris, CNRS, 1988.

DocCOr_ms = Valérie Neveu, Les plus anciennes chartes en langue française conservées dans le département de la Côte-d'Or (1239-1270), thèse de l'École des Chartes, 1988.

DocDo = Monique Mestayer/Thomas Brunner, Les plus anciens documents en français conservés à Douai, ed. ms. par Monique Mestayer, saisie informatique sous la direction de Martin Glessgen et de Benoît Tock, préparation de l'édition électronique par Thomas Brunner, 2014.

DocDo_ms = Monique Mestayer, Chartes de Douai, ca 1970.

DocDoub-1_ms = Martine Lefèvre, Les plus anciennes chartes en langue française conservées dans le département du Doubs (1233-1261), thèse de l'École des Chartes, 1975.

DocDoub-2_ms = Judith Ducourtieux, Les plus anciennes chartes en langue française conservées aux Archives départementales du Doubs (1260-1271), thèse de l'École des Chartes, 1994 [revue par Anne-Caroline Belmon-Beaugendre].

DocFl = Reine Mantou, Documents linguistiques de la Belgique romane, vol. 1, Chartes en langue française antérieures à 1271 conservées en Flandre orientale et Flandre occidentale, Paris, CNRS, 1987.

DocFor = Marguerite Gonon, Documents linguistiques de la France (série francoprovençale), vol. 1 : Documents linguistiques du Forez (1260-1498), Paris, CNRS, 1974.

DocHain = Pierre Ruelle, Documents linguistiques de la Belgique romane, vol. 2 : Chartes en langue française antérieures à 1271 conservées dans la province de Hainaut, Paris, CNRS, 1984. 
DocHM = Jean-Gabriel Gigot, Documents linguistiques de la France (série française), vol. 1 : Chartes en langue française antérieures à 1271 conservées dans le département de la Haute-Marne, Paris, CNRS, 1974. - Saisie informatique sous la direction de Benoît Tock, édition électronique préparée par Dumitru Kihaï, DocLing-1 et -2.

DocHS = Claire Muller, Les plus anciens documents en français conservés dans le département de la Haute-Saône (1242-1300), Édition électronique, DocLing-2 [cf. DocJu].

Doc]u = Claire Muller, Les plus anciens documents en français conservés dans le département du Jura (1243-1296), Édition électronique, DocLing-2.

[L'édition repose sur la thèse de C.M., Médialité de la charte : caractérisation structurelle du genre textuel dans un corpus de chartes françaises inédites du XIII' siècle. Accompagné d'une édition de 230 chartes inédites, Université de Zurich, 2011]

DocJuBe $=$ Ernest Schüle/Rémy Scheurer/Zygmunt Marzys, Documents linguistiques de la Suisse Romande, vol. 1 : Documents en langue française antérieurs à la fin du XVI siècle conservés dans les cantons du Jura et de Berne, Paris, CNRS, 2002.

DocLing-1 = Les plus anciens documents linguistiques de la France. Édition électronique, Collection fondée par Jacques Monfrin, poursuivie par Martin Glessgen, première édition en collaboration avec Françoise Vielliard et Olivier Guyotjeannin (12009).

[= édition et reproduction photographique de 1.133 documents français originaux (1224-1275) conservés dans les départements de la Marne, de la Haute-Marne, de la Meurthe-et-Moselle et de la Meuse (= DocMa, DocHM, DocMM, DocMe)]

DocLing-2 = Les plus anciens documents linguistiques de la France (DocLing). Édition électronique, dirigée par Martin Glessgen, en partenariat avec Frédéric Duval et Paul Videsott. Deuxième édition entièrement revue et élargie ( $\left.{ }^{2} 2014\right)<w w w . r o s e . u z h . c h / d o c l i n g>$.

[= édition et reproduction photographique de 2.185 documents français originaux (1204-1331), provenant du Nord-Est de la France (Flandre, Champagne, Lorraine, Franche-Comté, Bourgogne) et de la chancellerie Royale (= DocDo, Doc)u, DocHM, DocHS, DocMa, DocMe, DocMM, DocNi, DocR, DocSL, DocV)]

DocLyo = Paulette Durdilly, Documents linguistiques de la France (série francoprovençale), vol. 2 : Documents linguistiques du Lyonnais (1225-1425), Paris, CNRS, 1975.

DocMa = Dumitru Kihaï, Les plus anciens documents en français conservés dans le département de la Marne (1234-1272), Édition électronique, DocLing-1 et -2.

[L'édition repose sur la thèse de D. K., Écriture et pouvoir au $13^{e}$ siècle en Champagne, Zurich/ Strasbourg, 2011]

DocMe $=$ Anne-Christelle Matthey, Les plus anciens documents en français conservés dans le département de la Meuse (1225-1270), Édition électronique, DocLing-1 et -2.

[L'édition repose sur la thèse d'A.-C. M., Les plus anciens documents linguistiques de la France : le cas du département de la Meuse, Zurich, 2008]

DocMM = Martin Glessgen/Michel Arnod, Les plus anciens documents en français conservés dans le département de la Meurthe-et-Moselle (1232-1265), ed. ms. par M. Arnod, révision, édition électronique intégralement réélaborée par M. Glessgen, DocLing-1 et - 2 .

DocMM_ms = Michel Arnod, Publication des plus anciennes chartes en langue vulgaire antérieures à 1265 conservées dans le département de Meurthe-et-Moselle, Thèse de $3^{\mathrm{e}}$ cycle dactylographiée, Nancy 1974.

DocNeu = Morerod, Jean-Daniel, et al., Documents linguistiques de la Suisse Romande, vol. 2 : Documents conservés dans le canton de Neuchâtel, en préparation.

Doc $\mathrm{Ni}=$ Julia Alletsgruber, Les plus anciens documents en français conservés dans le département de la Nièvre (1289-1330), Édition électronique, DocLing-2.

[L'édition repose sur la thèse de J. A., Étude du lexique de l'agriculture dans des textes documentaires français du XIII siècle, Zurich/Paris, 2012] 
DocOis = Louis Carolus-Barré, Les plus anciennes chartes en langue française, t. 1 : Problèmes généraux et recueil des pièces originales conservées aux Archives de l'Oise (1241-1286), Paris, Klincksieck, 1964. [202 docs]

DocPC_ms = Pierre Bougard, Chartes du Pas-de-Calais, ca 1980.

DocPoit = Milan S. La Du, Chartes et documents poitevins du XIII siècle en langue vulgaire, 2 vol., Poitiers, 1960/1964.

DocR = Paul Videsott, Les plus anciens documents en français de la chancellerie royale capétienne (1241-1300), Édition électronique, DocLing-2. - Édition papier, Strasbourg, ÉLiPhi, 2015.

DocSL = Julia Alletsgruber, Les plus anciens documents en français conservés dans le département de la Saône-et-Loire (1227-1331), Édition électronique, DocLing-2 [cf. DocNi].

DocSom_ms = Jean Estienne, Chartes de la Somme, ca 1980.

DocV = Jean Lanher, Documents linguistiques de la France (série française), vol. 2 : Chartes en langue française antérieures à 1271 conservées dans le département des Vosges, Paris, CNRS, 1975. - Édition électronique par David Trotter, 2014.

Lodge, Anthony (2006, 2010), Les comptes des consuls de Montferrand (1273-1319, 1346-1373), 2 vols., Paris, École des Chartes. - Édition électronique du premier vol. <http://elec.enc. sorbonne.fr/montferrand>.

Meyer, Paul (1874/1876), Recueil d'anciens textes bas-latins, provençaux et français, 2 vol., Paris, Librairie Franck.

Meyer, Paul (1891), Le langage de Die au XIII siècle, Romania 20, 70-85.

Meyer, Paul (1898a), Documents linguistiques des Basses-Alpes, Romania 27, 337-441.

Meyer, Paul (1898b), Le livre-journal de maître Ugo Teralh : notaire et drapier à Forcalquier (1330-1332), in : Notices et extraits des manuscrits de la Bibliothèque Nationale et autres bibliothèques, 36, 129-170.

Meyer, Paul (1906), Fragments du grand livre d'un drapier de Lyon (1320-1323), Romania 35, 428-444. Meyer, Paul (1909), Documents linguistiques du Midi de la France, recueillis et publiés avec glossaires et cartes. Ain, Basses-Alpes, Hautes-Alpes, Alpes-Maritimes, Paris, Champion.

Ourliac, Paul/Magnou, Anne-Marie (1985), Le cartulaire de la Selve. La terre, les hommes et le pouvoir en Rouergue au XII siècle, Paris, CNRS, 1985.

\section{2 Études}

Belmont, Jérôme/Vielliard, Françoise (1997), Latin farci et occitan dans les actes du XI siècle, Bibliothèque de l'École des Chartes 155, 149-183.

Brunel, Clovis (1922), Les premiers exemples de l'emploi du provençal dans les chartes, Romania 48, 335-364.

Brunner, Thomas (2014), Douai, une ville dans la révolution de l'écrit du XIII siècle, thèse ms., Strasbourg.

Carles, Hélène (s.p.), Trésor galloroman des origines (TGO). La trajectoire étymologique et la variation géolinguistique du lexique galloroman présent dans les actes latins originaux (ca 800-1120), Strasbourg, ÉLiPhi.

Carles, Hélène/Glessgen, Martin (2015), La philologie éditoriale et linguistique, in : Claudia PolzinHaumann/Wolfgang Schweickard/ (edd.), Manuel de linguistique française (Manuals of Romance Linguistics 8), Berlin/Boston, De Gruyter.

Chambon, Jean-Pierre (1980), Notes d'ancien auvergnat. En relisant le « testament » de Peironelle de Bulhon [Brunel 1926, $n^{\circ}$ 282], Romania 102, 226-237.

Chambon, Jean-Pierre (1987), Identification de noms de lieu des «Plus Anciennes Chartes en langue provençale », Bibliothèque de l’École des chartes 145, 193-202. 
Chambon, Jean-Pierre (2006), Lexicographie et philologie : réflexions sur les glossaires d'éditions de textes (français médiéval et préclassique, ancien occitan), Revue de Linguistique Romane 70, 123-141.

Chambon, Jean-Pierre (2012), Développement et problèmes actuels des études occitanes, Revue de Linguistique Romane 76, 199-210.

Chambon, Jean-Pierre/Olivier, Philippe (2000), L'histoire linguistique de l'Auvergne et du Velay: notes pour une synthèse provisoire, Travaux de Linguistique et de Philologie 38, 83-153.

Dees, Anthonij (1980), Atlas des formes et des constructions des chartes françaises du $13^{e}$ siècle, Tübingen, Niemeyer.

Dinguirard, Jean-Claude (1979), Observations sur le gascon des plus anciennes chartes, Via Domitia. Annales de la Faculté de Lettres de Toulouse N.S. 22, 9-46.

Dondaine, Colette (2002), Trésor étymologique des mots de la Franche-Comté, Strasbourg, SLR.

Drüppel, Christoph Josef (1984), Altfranzösische Urkunden und Lexikologie : Ein quellenkritischer Beitrag zum Wortschatz des frühen 13. Jahrhunderts, Tübingen, Niemeyer.

Gévaudan, Paul/Glessgen, Martin (s.p.), Le manuscrit performatif. Subordination et modalité dans les actes français $d u 13^{e}$ siècle, $\mathrm{ms}$.

Glessgen, Martin (1997), Compte rendu de Hug-Mander 1989, Zeitschrift für romanische Philologie 113, 89-93.

Glessgen, Martin (2001a), Das altfranzösische Geschäftsschrifttum in Oberlothringen : Quellenlage und Deutungsansätze, in : Kurt Gärtner et al. (edd.), Skripta, Schreiblandschaften und Standardisierungstendenzen. Beiträge zum Kolloquium vom 16. bis 18. September 1998 in Trier, Trier, THF, 257-294.

Glessgen, Martin (2001b), L'élaboration philologique et l'étude lexicologique des «Plus anciens documents linguistiques de la France » à l'aide de l'informatique, in : Frédéric Duval (ed.), Frédéric Godefroy. Actes du Xe colloque international sur le moyen français, Paris, École des Chartes, 2003, 371-386.

Glessgen, Martin (2004), Realia und Urkunden. Die Teilung eines lothringischen Stadthauses kurz nach 1400, in : Alberto Gil et al. (edd.), Romanische Sprachwissenschaft. Zeugnisse für Vielfalt und Profil eines Faches. Festschrift für Christian Schmitt zum 60. Geburtstag, Frankfurt a.M. et al., Lang, 423-447.

Glessgen, Martin (2007), Philologie und Sprachgeschichtsschreibung in der Romanistik : Die « informatische Wende », in : Matthias Stolz (ed.), Edition und Sprachgeschichte. Baseler Fachtagung 2.-4. März 2005, Tübingen, Niemeyer, 201-212.

Glessgen, Martin (2008), Les lieux d'écriture dans les chartes lorraines du XIII' siècle, Revue de Linguistique Romane 72, 413-540.

Glessgen, Martin (2011a), L'élaboration philologique et linguistique des «Plus anciens documents linguistiques de la France, Édition électronique », trois études réunies par Martin Glessgen, Dumitru Kihaï et Paul Videsott, Bibliothèque de l'École des Chartes 168 (2010) [2011], 5-94 [pp. 7-24 : Id., Présentation générale : Architecture et méthodologie du projet des Plus anciens documents linguistiques de la France, Édition électronique »].

Glessgen, Martin (2011b), Le long chemin d'une charte vers l'ère multimédiale, in : Anja Overbeck/ Wolfgang Schweickard/Harald Völker (edd.), Lexikon, Varietät, Philologie, Romanistische Studien. Günter Holtus zum 65. Geburtstag, Berlin/New York, De Gruyter, 649-664.

Glessgen, Martin (2012), Trajectoires et perspectives en scriptologie galloromane, Medioevo Romanzo 36, 5-23.

Glessgen, Martin/Tittel, Sabine (2015), Les «Plus anciens documents linguistiques de la France » et le DEAFélectronique, Revue de Linguistique Romane 79 (sous presse).

Goebl, Hans (1970), Die normandische Urkundensprache. Ein Beitrag zur Kenntnis der nordfranzösischen Urkundensprachen des Mittelalters, Wien, Österreichische Akademie der Wissenschaften. 
Gossen, Carl Theodor (1967), Französische Skriptastudien. Untersuchungen zu den nordfranzösischen Urkundensprachen des Mittelalters, Wien, Österreichische Akademie der Wissenschaften.

Grafström, Åke (1958), Étude sur la graphie des plus anciennes chartes languedociennes avec un essai d'interprétation phonétique, Uppsala, Almqvist \& Wiksell.

Grafström, Åke (1968), Étude sur la morphologie des plus anciennes chartes languedociennes, Stockholm, Almqvist \& Wiksell, 1968.

Grafström, Åke (1978), Quelques observations sur les plus anciennes chartes limousines, Via Domitia. Annales de la Faculté de Lettres de Toulouse N.S. 14, 217-229 [= Hommage à Jean Séguy vol. 1].

Grübl, Klaus (2013), Les travaux philologiques de Louis Carolus-Barré, in : Bruno Ricard (ed.), Histoire médiévale, érudition et recherche dans l'Oise. Hommage à Louis Carolus-Barré, 30-35 (http:// archives.oise.fr/action-culturelle/publications/actes-de-colloque-et-autres-publications/, consulté le 22 août 2014).

Grübl, Klaus (2014), Varietätenkontakt und Standardisierung im mittelalterlichen Französisch. Theorie, Forschungsgeschichte und Untersuchung eines Urkundenkorpus aus Beauvais (1241-1455), Tübingen, Narr.

Hayez, Jérôme (2005), L'Archivio Datini, de l'invention de 1870 à l'exploration d'un système d'écrits privés, Mélanges de l'École française de Rome. Moyen Âge 117, 121-191.

Höfler, Manfred (1967), Untersuchungen zur Tuch- und Stoffbenennung in der französischen Urkundensprache, Tübingen, Niemeyer.

Holtus, Günter/Overbeck, Anja/Völker, Harald (2003), Luxemburgische Skriptastudien. Edition und Untersuchung der altfranzösischen Urkunden Gräfin Ermesindes (1226-1247) und Graf Heinrichs V. (1247-1281) von Luxemburg, Tübingen, Niemeyer.

HSK 10 = Günther, Hartmut/Ludwig, Otto (edd.), Schrift und Schriftlichkeit / Writing and Its Use, 2 vols., Berlin/New York, De Gruyter, 1994.

Hug-Mander, Angela (1989), Die okzitanischen Urkunden im Departement Alpes-de-Haute-Provence. Untersuchung einiger graphischer, phonetischer und morphologischer Erscheinungen, Bern et al., Lang.

InvSyst = Barbara Frank/Jörg Hartmann (1997), Inventaire systématique des premiers documents des langues romanes, 5 vols., Tübingen, Narr.

Kabatek, Johannes (2005), Die Bolognesische Renaissance und der Ausbau romanischer Sprachen. Juristische Diskurstraditionen und Sprachentwicklung in Südfrankreich und Spanien im 12. und 13. Jahrhundert, Tübingen, Niemeyer.

Kalman, Hans (1974), Étude sur la graphie et la phonétique des plus anciennes chartes rouergates, Phil. Diss. Zürich.

Koch, Peter (1987), Distanz im Dictamen. Zur Schriftlichkeit und Pragmatik mittelalterlicher Brief- und Redemodelle in Italien, Freiburg i.Br., ms.

Koch, Peter (1990), Von Frater Semeno zum Bojaren Neacşu. Listen als Domäne früh verschrifteter Volkssprache in der Romania, in : Wolfgang Raible (ed.), Erscheinungsformen kultureller Prozesse, Tübingen, Narr, 121-165.

Krefeld, Thomas (1985), Das französische Gerichtsurteil in linguistischer Sicht : Zwischen Fach- und Standessprache, Frankfurt et al., Lang.

Linder, Karl Peter (1970), Studien zur Verbalsyntax der ältesten provenzalischen Urkunden und einiger anderer Texte mit einem Anhang über das konditionale QUI, Tübingen, Fotodruck.

Mazziotta, Nicolas (2009), Ponctuation et syntaxe dans la langue française médiévale. Étude d'un corpus de chartes originales écrites à Liège entre 1236 et 1291, Tübingen, Niemeyer.

Melis, Federigo (1962), Aspetti della vita economica medievale : studi nell'archivio Datini di Prato, 2 vol., Siena, Monte dei paschi di Siena.

Monfrin, Jacques (1968), Le mode de tradition des actes écrits et les études de dialectologie, Revue de Linguistique Romane 32, 17-47 [réimpression in : Georges Straka (ed.), Les dialectes de France 
au Moyen Age et aujourd'hui. Colloque organisé ... du 22 au 25 mai 1967, Paris, Klincksieck, 1972, 25-55].

Monfrin, Jacques (1974), Introduction [= Les études sur les anciens textes gallo-romans non littéraires ; Le recueil des documents linguistiques de la France etc.], in : DocHMG [1974], XI-LXXX.

Olivier, Philippe (2009), Dictionnaire d'ancien occitan auvergnat. Mauriacois et Sanflorain (1340-1540), Tübingen, Niemeyer.

Pfister, Max (1970), Lexikalische Untersuchungen zu Girart de Roussillon, Tübingen, Niemeyer.

Schroeder, Walter (1932), Die bedingte Diphthongierung betonter Vokale im südfranzösischen Alpengebiet, Volkstum und Kultur der Romanen 5, 152-141 [reprend la thèse de l'auteur, Hamburg 1928].

Soutou, André (1976), Remarques sur une chartes rouergates de 1161, Via Domitia. Annales de la Faculté de Lettres de Toulouse N.S. 12, 95-99.

Trotter, David (2015), Coup d'œil sur les scriptae médiévales et les textes qui les représentent, in : Maria Ilescu/Eugeen Roegiest (edd.), Manuels des anthologies, corpus et textes romans (Manuals of Romance Linguistics 7), Berlin/Boston, De Gruyter.

Videsott, Paul (2009), Padania scrittologica. Analisi scrittologiche e scrittometriche in testi in italiano settentrionale antico dalle origini al 1525, Tübingen, Niemeyer.

Videsott, Paul (2013), Les débuts du français à la Chancellerie royale : analyse scriptologique des chartes de Philippe III (1270-1285), Revue de Linguistique Romane 77, 3-49. 